\title{
Oral lichenoid lesion and proximity to restoration
}

\author{
Oral lichenoid lesions related to dental restorative materials
}

\section{Y. Issa, A. J. Duxbury, T. V. Macfarlane and P. A. Brunton Br Dent J 2005; 198: 361-366}

Objective

To determine the effectiveness of replacing restorations considered to be the cause of an oral lichenoid lesion (oral lichenoid reaction)(OLL).

\section{Design}

Clinical intervention and nine-month follow up.

Setting

The study was carried out in the University Dental Hospital of Manchester, 1998-2002.

\section{Subjects and methods}

A total of 51 patients, mean age 53 (SD 13) years, who had oral lesions or symptoms suspected to be related to their dental restorations were investigated. Baseline patch tests for a series of dental materials, biopsies and photographs were undertaken. Thirty-nine out of 51 patients (76\%) had their restorations replaced.

\section{Results}

The clinical manifestations of OLL were variable; the majority of OLL were found to be in the molar and retro molar area of the buccal mucosa and the tongue. Twenty-seven (53\%) patients had positive patch test reactions to at least one material, 24 of them for one or more mercury compound. After a mean follow up period of nine months, lesions adjacent to replaced restorations completely healed in $16(42 \%)$ patients (10 positive and 6 negative patch tests). Improvement in signs and symptoms were found in 18 (47\%) patients (11 positive and 7 negative patch tests).

\section{Conclusion}

OLLs may be elicited by some dental restorations. Replacing restorations adjacent to these lesions is associated with healing in the majority of cases particularly when lesions are in close contact with restorations. A patch test seems to be of limited benefit as a predictor of such reactions.

\section{IN BRIEF}

- Oral lichenoid lesions (OLLs) may be elicited by dental restorations and amalgam restorations with their mercury content appear to be a major aetiological factor

- The diagnosis depends mainly on the clinical findings including the lesions' characteristics and relationship to restorations. Patch testing seems to be of limited value.

- Replacement of dental restorations can result in the resolution or improvement of OLLs in most instances.

\section{COMMENT}

This study attempted to define the clinical features of amalgam related oral lichenoid lesions (OLL) and to evaluate the effectiveness of replacing or isolating (crowns) amalgam restorations adjacent to OLL. In addition the authors also attempted to evaluate the accuracy of patch testing as a clinical indicator for amalgam replacement.

The clinical and histopathological features of oral lichen planus (OLP) and oral lichenoid lesions (OLL) are almost identical. Oral pathologists will agree it is extremely difficult, if not impossible to differentiate OLP from OLL on a histopathological basis.

According to this study, significant improvement (complete healing $-42 \%$ ) has been achieved in $89 \%$ of patients. It is also interesting to note from this study that a simple straightforward clinical assessment involving the characteristics of the lesion and its proximity to an amalgam restoration would be a better predictor and prognostic marker than other available methods. This finding would encourage general dental practitioners (GDPs) to manage patients with amalgam related OLL effectively at the primary care setting in the future.

At present it is difficult for GDPs to get approval for amalgam replacement under the NHS, especially when the patch test result is negative. The patients most affected by this arrangement are those exempted from NHS payment. However according to this study, $81 \%$ of the patients with negative patch test results showed complete healing or significant improvement following amalgam replacement.

These findings should be taken into consideration when new guidelines are formatted so that the patients, especially those that fall into the lower economic social net will not be affected. As shown in this study a combination of topographical proximity of OLL and a restoration and a confirmation of a histopathological report of lichenoid mucositis would be a sufficient indicator for amalgam replacement rather than relying on the accuracy of patch testing for NHS approval.

Thirty-four patients were involved in the study and were followed up for a mean period of nine months. Composite materials used in these patients have only a short follow up history compared to amalgam. A long-term follow up with more patients is needed on these materials in order to properly evaluate toxicity, allergy and irritability in general and especially towards the oral mucous membrane. As shown in the conclusion, further laboratory and clinical studies are needed to achieve a clear understanding of OLL and OLP and to provide clear diagnostic and prognostic markers with high specificity and sensitivity.

S. Ariyaratnam, Specialist in Oral Medicine and Surgical Dentistry and Clinical teacher in Oral Medicine,

Oral Medicine unit, Dental Hospital, Manchester doi:10.1038/sj.bdj.4812183 